\title{
A New Approach based on Brownian Motion for the Simulation of Ultra-Small Semiconductor Devices
}

\author{
CLINTON R. AROKIANATHAN, ASEN ASENOV and JOHN H. DAVIES \\ Department of Electronics and Electrical Engineering, Glasgow University, Glasgow, G12 8QQ, U.K.

\begin{abstract}
We present a new approach to the simulation of ultra-small semiconductor devices based on Brownian motion of the carriers described by the Langevin equation. It follows the trajectories of individual particles in real space but does not require the computational effort of a full Monte Carlo simulation. This method is particulary useful for modeling very small devices where individual impurities and carries must be considered as discrete entities, and where a molecular dynamics approach is used in a full-scale three-dimensional simulation. We show that this method gives the correct Maxwell-Boltzmann distribution and that it provides a good description of transport through a short diode.
\end{abstract}

Keywords: Brownian Dynamics, submicron, fluctuations, short diode

\section{INTRODUCTION}

As a result of the progressive miniaturisation of components in integrated circuits, the quantised nature of the charges emerge. At these length scales, it becomes no longer accurate to represent them by continuous distribution functions and the random fluctuations in the densities between different regions of an individual device begin to have a significant effect on its characteristics [1-2]. This necessitates a full 3-dimensional simulation of these systems, requiring sizeable computational resources, so a suitable method must be found which reduces this burden as much as possible. These considerations favour methods which follow the motion of individual particles over those which rely on the solution of partial differential equations. The use of Brownian dynamics offers a realistic alternative where the full apparatus of the Monte Carlo techniques are not essential, especially in simulations involving low fields and high particle densities.
We shall first discuss the Langevin theory [3] and its discrete time approximation. Then the practical implementation of the Brownian dynamics approach is outlined. Finally, we test our approach in a simulation of a p-n junction diode. The results of this test are in excellent agreement both with the ideal diode equation and with the drift-diffusion results obtained from the commercial simulator MEDICI.

\section{THE LANGEVIN EQUATION AND ITS DISCRETE TIME APPROXIMATION.}

The Brownian simulation approach described here is based on the Langevin equation,

$$
m \dot{\mathbf{v}}=q \mathbf{E}-\frac{m \mathbf{v}}{\tau}+\mathbf{F}(t) .
$$

The complicated scattering mechanisms have been greatly simplified in the Langevin approach. A frictional force models the dissipative effects of scatter- 
ing through the momentum relaxation time $\tau$, and a random fluctuating force $\mathbf{F}(t)$ represents the stochastic kicks received by the particle from the phonon bath. The balance between the frictional and the fluctuating forces leads to a Boltzmann distribution of velocity in thermal equilibrium. An important feature of our approach is that the impurities and other carriers exert their influences on the motion of individual particle through modulations of the electric field $\mathbf{E}$. Thus $\mathbf{F}(t)$ represents only the phonon scattering.

The Langevin equation must be discretized for numerical simulation. The random force $\mathbf{F}(t)$ requires special treatment and has to be integrated separately,

$$
\Delta \mathbf{V}_{\text {fluc }}(t)=\frac{1}{m} \int_{t}^{t+\Delta t} \mathbf{F}\left(t^{\prime}\right) d t^{\prime}
$$

It is rapidly varying on any time scale because it is $\delta$ correlated and also averages to zero. The Wiener process $\Delta \mathbf{V}_{\text {fluc }}(t)$ follows a Gaussian distribution due to the central limit theorem. The associated diffusion coefficient $D_{v}$ in velocity space is related to $\tau$ through the fluctuation-dissipation theorem. The variance of the distribution of $\Delta \mathbf{V}$ fluc $(t)$ can therefore be written in the two forms

$$
\left\langle\Delta \mathbf{V}_{f l u c}^{2}(t)\right\rangle=2 D_{v} \Delta t=\frac{2 k_{B} T \Delta t}{\tau m}
$$

where $k_{B}$ is Boltzmann's constant and $T$ is the temperature. We can now integrate the Langevin equation to give a finite difference equation. Introducing a discrete time $t_{n}=n \Delta t$ and the notation $v_{n}=v(n \Delta t)$ we have

$$
\mathbf{v}_{n+1}=\gamma \mathbf{v}_{n}+\frac{q}{m} \mathbf{E} \Delta t+\Delta \mathbf{v}_{n}
$$

where $\mathbf{E}_{n}=\mathbf{E}\left(t_{n}\right)$ and $\gamma=1-\Delta t / \tau$. We shall now consider the practical implementation of this scheme.

\section{PRACTICAL IMPLEMENTATION.}

The implementation of the Brownian simulation approach based on the discrete time approximation of the Langevin equation is straightforward. The only input parameters required are the mobilities and effective masses of the carriers and the lattice temperature, as in the drift-diffusion method. The procedures involved in the simulation are very similar to those used in the ensemble Monte Carlo approach [4,5].

The time step $(\Delta t)$ must be smaller that $\tau$ to guarantee convergence. The components of the random velocity $\Delta \mathbf{V}_{n}$ can be randomly chosen from a Gaussian distribution of variance $2 D_{1} \Delta t$. This gives the correct statistical distribution of $\Delta \mathbf{V}_{n}$ immediately. It may be easier, however, to use steps of constant magnitude $\sqrt{2 D_{v} \Delta t}$. This also results in the same Gaussian distribution after some time steps; the frictional term is helpful here since it smoothes the stochastic jumps. The simplicity of this approach pays great dividends when $\Delta t / \tau$ becomes small. It is convenient to use the leapfrog scheme to follow the motion of the particles [6].

\section{VERIFICATION}

A series of tests was performed to confirm the validity and accuracy of our Brownian simulation method. Figure 1shows the distribution of velocities at equilibrium. This is clearly in excellent agreement with a Maxwell distribution at the expected temperature of $300 \mathrm{~K}$. The distribution is shifted when a field of 1 $\mathrm{MV} \mathrm{m} \mathrm{m}^{-1}$ is applied. Note that the temperature of the electrons does not increase with electric field in this model. This is to be expected from its close relation to the drift-diffusion approximation.

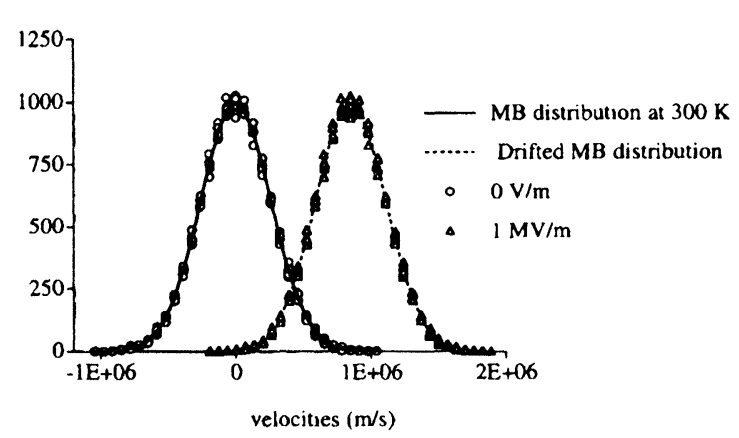

FIGURE 1 Distribution of velocities in equilibrium and under a constant electric field. Note that a field shifts the distribution without affecting its width 
A $p n$ junction diode provides an excellent test for any approach to simulation because both drift and diffusion are critical. At equilibrium, the width of the depletion region and the built in potential are established by the balance between drift and diffusion. At low forward bias the current is dominated by diffusion of the minority carriers away from the depletion layer, giving an exponential current-voltage characteristic (the Shockley equation). At high current level the series resistance and the drift current in the undepleted regions of the diode lead to a linear (Ohmic) behaviour. Hence the diode operation can be described satisfactory using drift-diffusion type simulations. The comparison of standard drift-diffusion simulations with the Brownian results for a simple diode will establish further the credibility of the new approach.

In our test we consider an $1 \mu \mathrm{m}$ long, symmetric $\mathrm{Si}$ diode with doping of $10^{22} \mathrm{~m}^{-3}$. Recombination was neglected since the length of the diode was much shorter than the minority carrier diffusion lengths on both sides of the depletion region. The results (figure 2) were compared with the Shockley equation for an ideal short diode and found to be in good agreement for small forward currents. The agreement between the Brownian approach and the MEDICI drift-diffusion simulation is good both at low and high current levels. This confirms that the new method properly describes the effects due to diffusion at low bias and those due to drift at higher applied voltages.

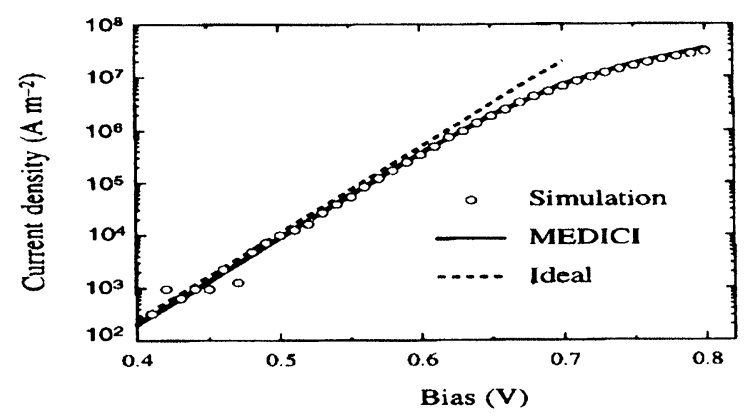

FIGURE 2. The $I(V)$ characteristics of a $1 \mu \mathrm{m}$ Si diode predicted by the Brownian model (open circles), the commercial drift-diffusion simulator MEDICI (solid line) and the Shockley model (broken line). The Brownian model works well in both the diffusion-dominated regime at low bias and when drift dominates at high bias
The statistical noise due to the quantisation of electronic charge is apparent during the small injection rates that are present when a small forward bias is applied. The very small discrepancy at large forward bias is due to insufficient injection rates at the contacts, which were modelled as reservoirs in thermal equilibrium with no external fields.

\section{CONCLUSION}

Brownian dynamics offers a simple solution to the modelling of small devices. It has particular uses where a drift-diffusion model would be adequate, but the particulate nature of the charge carriers is essential. It has some similarities to the Monte Carlo method using the relaxation time approximation but the large discontinuous changes in momenta in the latter are replaced by a continuous process. It therefore requires fewer flights to get a statistically acceptable set of data. Simulating a diode is a difficult test for particle-based simulations and we have demonstrated that the Brownian model works well for both drift and diffusion in this case.

\section{References}

[1] R. W. Keyes, "Effect of Randomness in the Distribution of Impurity Ions on FET Thresholds in Integrated Electronics", IEEE JSSC, 245 (1972).

[2] H. Wong and Y. Taur, "Three-Dimensional 'Atomistic' Simulation of Discrete Random Dopant Distribution Effects in Sub-0.I $\mu m$ MOSFET's", IEEE IEDM, 705 (1993).

[3] R. Serra el al, Introduction to the Physics of Complex Systems, Oxford: Pergamon Press, 1986.

[4] C. Jacoboni and L. Reggiani, "The Monte Carlo method for the solution of charge transport in semiconductors with applications to covalent materials", Rev. Mod. Phys. 55, 645 (1993).

[5] C. Jacoboni and P. Lugli, The Monte Carlo Method for Semiconductor Device Simulation, Wein-New York : SpringerVerlag, 1989.

[6] R. W. Hockney and J. W. Eastwood, Computer Simulation Using Particles, New York : McGraw-Hill, 1981.

\section{Biographies}

Clinton R. Arokianathan graduated from Glasgow University in 1993 with a B. Eng. in Physics and Electronic Engineering. He is currently a postgraduate student at the Department of Electronics and Electrical Engineering. 
Asen Asenov (GU) had 10 years industrial experience as a head of the Process and Device Modelling Group in IME - Sofia. In 1985 he developed one of the first integrated process and device CMOS simulators IMPEDANCE. In 1989 - 1991 he was a visiting professor at the Physics Department of TU Munich. He joined the Department of Electronics and Electrical Engineering at Glasgow University in 1991. As a leader of the Device Modelling Group he has a major contribution in the development of the $2 \mathrm{D}$ and $3 \mathrm{D}$ device simulators and their application in the design of compound FETs, SiGe MOSFETs and IGBTs. He is working also on the design and performance of parallel algorithms.

John H. Davies has been a lecturer in Electronics and Electrical Engineering at Glasgow University since 1986. He has also worked at the University of California (Santa Barbara), Ohio State University, Cornell University, ETH-Zürich and AT\&T Bell Laboratories. His research on ultra-small semiconducting structures has been concerned particularly with the effect of doping fluctuations and the behaviour of donors and surfaces at low temperature. 

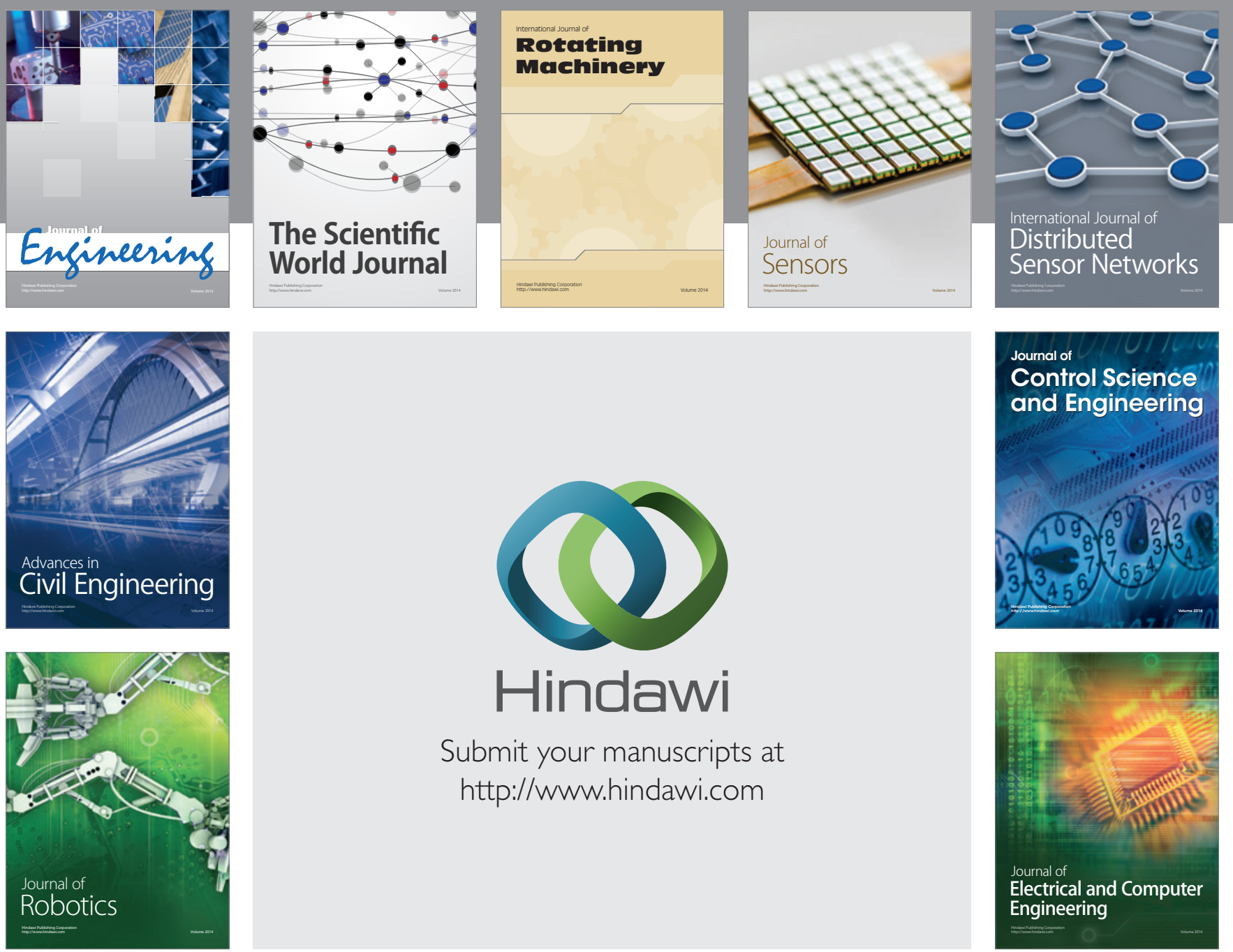

Submit your manuscripts at

http://www.hindawi.com
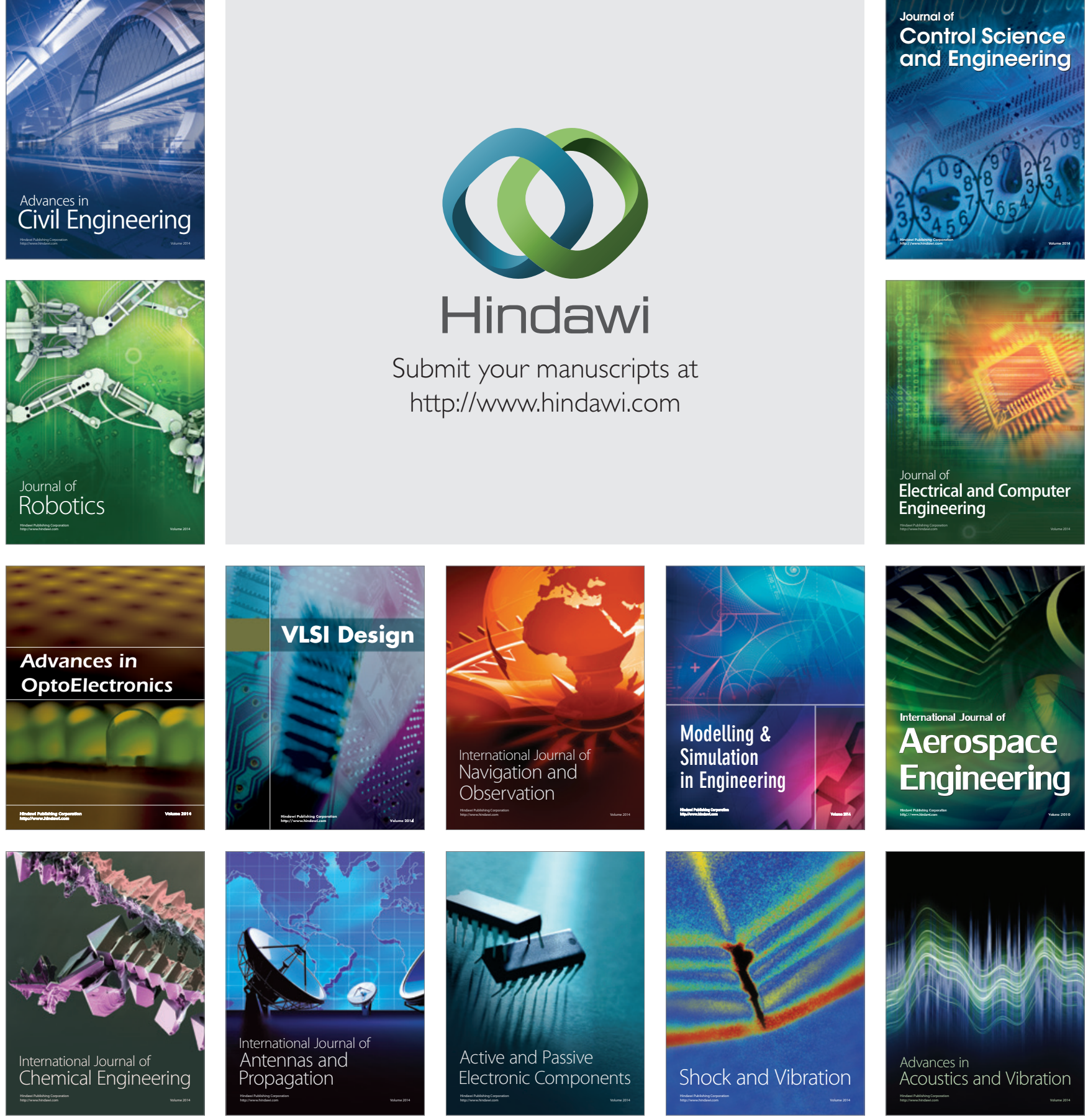